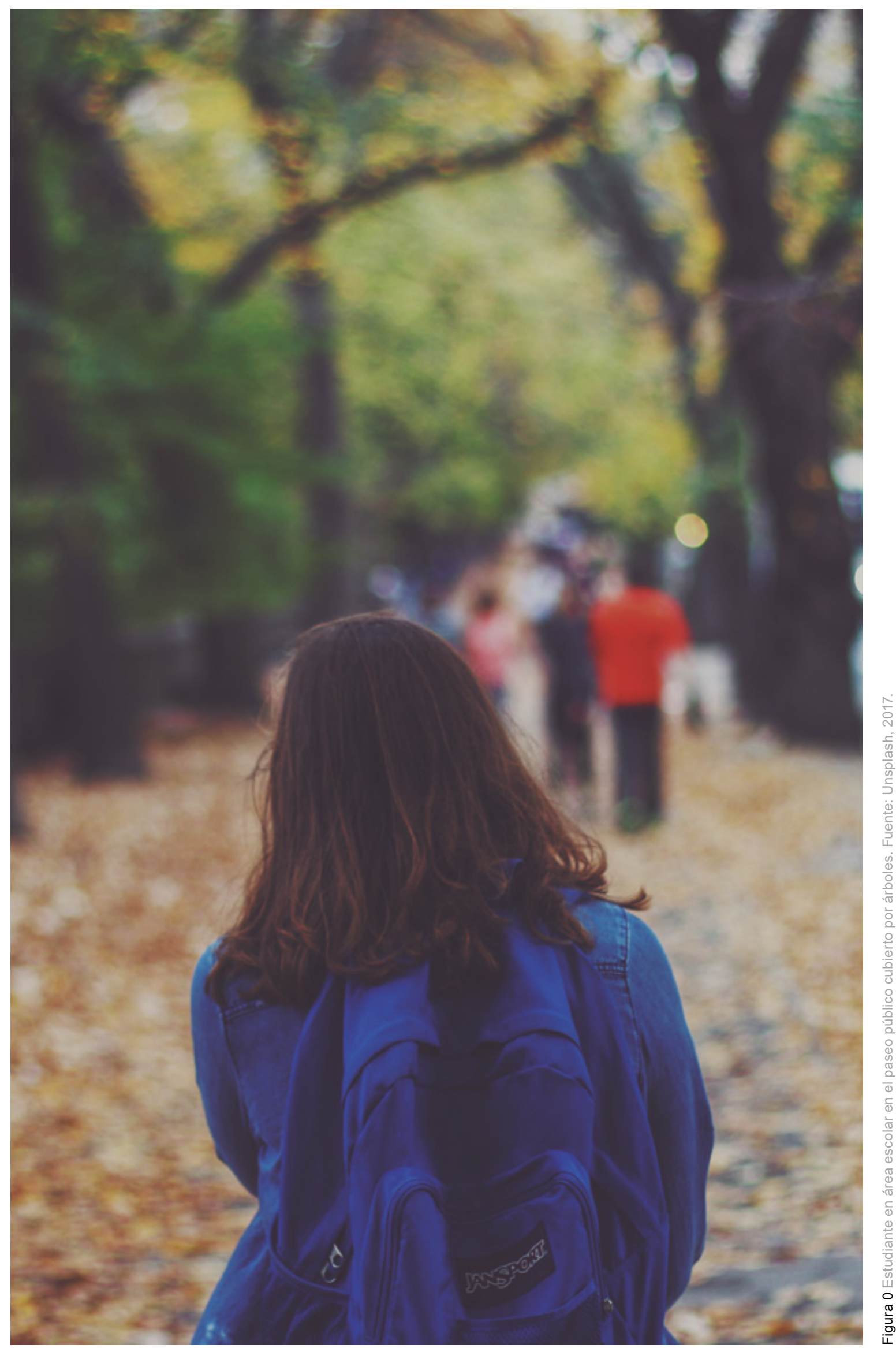

Arquitecturas del Sur / Vol 35 / No 52 / 2017 / ISSN Impresa 0716-2677 / ISSN Digital 0719-6466 Aprendiendo entre la naturaleza: Una revisión de los beneficios de los espacios verdes en el

96 Marina Bernardes,

Marina Bernardes, Lizandra Garcia Lupi Vergara / p. 96-103 

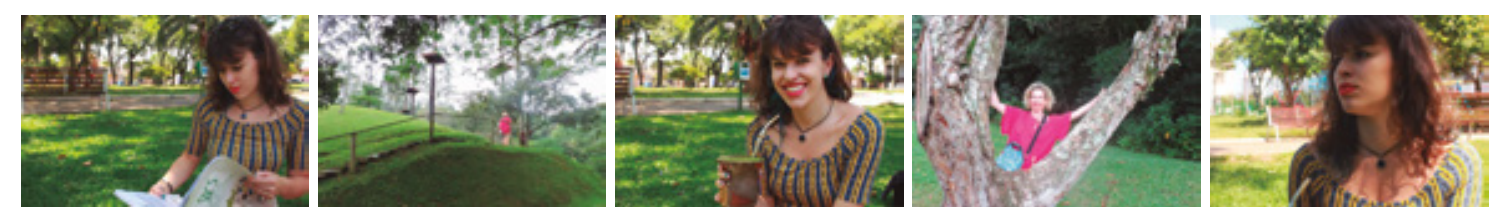

\title{
APRENDIENDO ENTRE LA NATURALEZA: UNA REVISIÓN DE LOS BENEFICIOS DE LOS ESPACIOS VERDES EN EL AMBIENTE ESCOLAR ${ }^{1}$
}

\author{
LEARNING IN NATURE: A REVIEW OF THE BENEFITS OF GREEN SPACE IN THE SCHOOL \\ ENVIRONMENT'
}

\section{Marina Bernardes², Lizandra Garcia Lupi Vergara ${ }^{3}$}

\section{RESUMEN}

Los problemas del aprendizaje encuentran interfaces en diversas áreas como lo es el espacio escolar. Este estudio investigó la literatura existente sobre el tema de los ambientes restauradores y la teoría de la restauración de la atención, en relación al ambiente escolar. No hay estudios sobre estas problemáticas en los ambientes escolares de América del Sur. El método empleado en este trabajo fue una revisión sistemática de la literatura, en el idioma inglés, para luego analizar los estudios aplicados y los resultados ya obtenidos. Los resultados presentan una gama de investigaciones que identifican los beneficios de los espacios verdes para la restauración de la atención de los estudiantes. Desde esta perspectiva, fue posible determinar la importancia de la exposición a ambientes naturales para favorecer el desempeño estudiantil de las futuras poblaciones académicas, así como también resaltar la importancia de desarrollar estudios en esta área, considerando los efectos positivos que generan las áreas verdes en el espacio escolar.

Palabras clave: arquitectura, escuela, ambiente educacional, atención, vegetación.

\begin{abstract}
Learning difficulties have interfaces in various areas such as school space. This study investigated the existing literature on the subject of restorative environments and the theory of attention restoration relative to the school environment. There are no studies about whether this effect occurs in South American school environments. The method employed in this research was a systematic review of literature in the English language to analyze the applied studies and the results obtained. The results present a range of research that identifies the benefits of green space for the restoration of student attention. From this perspective, it was possible to determine the importance of exposure to natural environments to favor the student achievement of future academic populations, as well as highlight the importance of carrying out studies in this area considering the positive effects of green areas in school space.
\end{abstract}

Keywords: architecture, school, educational environment, attention, vegetation.

Artículo recibido el 8 de agosto de 2017 y aceptado el 23 de diciembre de 2017 DOI: https://doi.org/10.22320/07196466.2017.35.052.09

[1] Este artículo está basado en los resultados de investigación de la tésis de magister en desarrollo del programa de Posgrado en Arquitectura y Urbanismo de la Universidad Federal de Santa Catarina (UFSC) titulada: Investigación en ergonomía: configuración arquitectónica de aulas como ambientes restauradores, financiada por BECA CAPES -DS.

[2] Universidad Federal Santa Catarina, Florianápolis, Brasil.marina.bernardes@posgrad.ufsc.br

[3] Universidad Federal Santa Catarina, Florianápolis, Brasil. I.vergara@ufsc.br 


\section{INTRODUCCIÓN}

La arquitectura ofrece soluciones a los espacios, examinando la calidad de los ambientes proyectados, y en lo que se refiere al bienestar de los usuarios, ha vuelto su foco principalmente a las adecuaciones físicas, como por ejemplo, construir un ambiente accesible. Sin embargo, pocos estudios se centran en aspectos cognitivos involucrados, al momento de diseñar un ambiente construido. El ambiente escolar es el lugar donde el niño pasa la mayor parte de su día, y uno de los principales aspectos al ser considerados durante la concepción de este ambiente es la ergonomía (Jayaratne, 2012). La falta de consideración de los aspectos ergonómicos, en sus dominios tanto físicos, cognitivos y organizacionales, puede comprometer no sólo la salud del alumno sino también su desempeño escolar.

El desempeño escolar depende de innumerables factores, como características de la escuela (físicas, pedagógicas, cualificación del profesor), de la familia (nivel de escolaridad de los padres, participación de los padres en la escuela y deberes) y del propio alumno. Se destaca, por lo tanto, que el problema de la dificultad de aprendizaje encuentra interfaces en diversas áreas, necesitando la atención de múltiples profesionales en diversos ámbitos de investigación (Araújo, 2002).

Siendo así, es necesario repensar el espacio escolar, pasando a considerar la diversidad de los niños, ya que la simple incomodidad de una silla puede perjudicar la atención en el desempeño de las tareas de enseñanza-aprendizaje. Lo que significa que el propio ambiente puede interferir en este proceso, dificultando o ayudando a que los niños mantengan atención (Choi, van Merrienboer y Paas 2014). Además de la aplicación adecuada de los aspectos relacionados con los ámbitos de la ergonomía, una forma de contribuir al bienestar cognitivo de los usuarios es a través de la proposición de espacios diseñados de acuerdo con el concepto de ambientes restauradores.

La restauración es un medio para recuperar o renovar, recursos psicológicos, fisiológicos y sociales, de las capacidades disminuidas por los esfuerzos diarios, los cuales resultan en una fatiga mental. El proceso de restauración puede ser promovido o posibilitado a través de ambientes restauradores (Hartig, 2004). Un ambiente restablecedor es aquel que promueve y permite la restauración de los recursos de los individuos, ya sean biológicos, psicológicos o sociales, necesarios para una adaptación exitosa a las circunstancias actuales (Løndal, 2013).

La teoría de la restauración de la atención (ART) explicita el proceso por el cual los ambientes físicos propician la recuperación de la capacidad de atención dirigida, la cual se ve fragilizada durante varias actividades cotidianas (Kaplan y Kaplan, 1989; Kaplan, 1995). Para Bagot, Allen y Toukhsati (2015), por su parte, lo que los niños relatan y lo que realmente es restaurador, de acuerdo con las propuestas teóricas de la ART, debe ser explorado. Y sugiere, además, que futuras investigaciones debieran extender su enfoque más allá de los ambientes naturales (áreas abiertas) y, entonces, comparar la restauración con ambientes ya construidos.

Considerando los preceptos de los ambientes restauradores, presentados en la próxima sección, se evidencia la influencia del ambiente escolar para la recuperación de la atención del niño. Este estudio pretende investigar cuál es la relación entre la teoría de la restauración de la atención y el ambiente escolar, a través de una revisión bibliográfica sistemática y de una síntesis narrativa de los estudios encontrados sobre este tema. 
La Teoría de la Restauración de la Atención (Attention Restoration Theory - ART)

De acuerdo con la ART, la restauración de la atención dirigida ocurre a partir de la "distancia psicológica" de los contenidos mentales de rutina, de la mano de una sensación de "fascinación" (fascination = cognitive or physical), sin esfuerzo, sostenida en ambientes coherentemente ordenados y de alcance sustancial. El alejamiento (being away) implica posibilidades geográficas y/o psicológicas de estar lejos. Para que se dé un ambiente restaurador dicho ambiente no solo debe proporcionar esta distancia, sino que debe promover un cambio en nuestros pensamientos, relacionado a las actividades cotidianas. Otra condición es la "extensión" (extend = scope and connectedness): un ambiente que tenga un alcance suficiente para mantener la interacción sin provocar aburrimiento durante un período de tiempo. Es decir, la extensión se refiere a las propiedades de conectividad y alcance en un lugar determinado. Así también, la teoría considera la "compatibilidad" (compatibility = with purpose), que se refiere al encuentro entre las inclinaciones personales, los propósitos y el soporte del ambiente para determinadas actividades y las posibles acciones humanas en el ambiente. Kaplan y Kaplan (1989) argumentan, en efecto, que estos cuatro factores generalmente se mantienen en niveles elevados en ambientes naturales. Cada uno de los componentes teóricos aborda un aspecto diferente de lo que hace un entorno potencialmente restaurador.

¿Los ambientes naturales pueden contribuir más a la restauración fisiológica, emocional y de la atención que el ambiente urbano? Un enfoque para esta cuestión apunta a considerar las formas en que las configuraciones naturales son susceptibles de satisfacer cada uno de los cuatro requisitos para un entorno restaurador.

Fascinación: La naturaleza está dotada de objetos fascinantes, además de ofrecer muchos procesos que las personas encuentran absorbentes. Una gama de fascinaciones ofrecidas por el paisaje natural se vincula con lo "suave", como las nubes, las puestas del sol, la nieve, el movimiento de la brisa de las hojas en la brisa - estos alistan la atención con facilidad, de forma espontánea. Atender a estas necesidades de ese modo ocurre sin esfuerzo, y ellos dejan amplia oportunidad para pensar sobre otras cosas

Alejamiento: Las configuraciones naturales son a menudo los destinos preferidos para las oportunidades de restauración extendidas. El litoral, montañas, lagos, arroyos, bosques son todos lugares idílicos para "huir". Sin embargo, para muchas personas del medio urbano, la oportunidad de huir a esos destinos no es una opción. Pero la sensación de alejamiento no requiere que la configuración sea lejana. Los ambientes naturales fácilmente accesibles pueden ofrecer un recurso importante para descansar la atención dirigida.

Extensión: En el contexto antiguo de libertad, vivir libre en la selva, la extensión ocurría fácilmente. No obstante, la extensión no necesariamente involucra grandes extensiones de tierra. Un área relativamente pequeña puede proporcionar una sensación de extensión. Las sendas y caminos pueden ser diseñados para que se vean mucho más grandes. La reducción del área o espacio facilita otro dispositivo para conferir una sen- sación de estar en un mundo completamente diferente, aunque el área no se extienda. Un ejemplo de ello, son los jardines japoneses: otorgan el sentido de alcance, así como la conexión. La extensión también funciona en un nivel más conceptual, como los lugares que incluyen artefactos históricos pueden promover un sentido o ser enviados a ambientes pasados $\mathrm{y}$, por lo tanto, a una amplia variedad de lugares.

Compatibilidad: El ambiente natural se experimenta a un nivel elevado en lo que se refiere a la compatibilidad. Es como si hubiera una conexión entre el paisaje natural y la condición humana. Para muchas personas, el funcionamiento en el escenario natural parece requerir menos esfuerzo que actuar en configuraciones más "civilizadas", aunque tenga una familiaridad mucho mayor con áreas urbanas. La gente, comúnmente, se aproxima a áreas naturales con el propósito de que estos patrones rápidamente se encuentren, aumentando así la compatibilidad.

La aplicación de la ART a los ambientes infantiles no ha sido estudiada, de modo que se percibe aquí la necesidad de una investigación que identifique los elementos que contribuyen al potencial restaurador de los ambientes infantiles (Bagot, Allen y Toukhsati, 2015). La teoría de Kaplan y Kaplan enfatiza que, para garantizar el funcionamiento diario efectivo, los seres humanos necesitan mantener la claridad cognitiva, lo que requiere atención dirigida. Sin embargo, cada individuo tiene una cantidad limitada de atención que es agotada por el uso extensivo. En términos generales, la fatiga de la atención deriva en menor competencia y eficiencia del funcionamiento cognitivo de las personas (Hartig, Mang y Evans, 1991).

Los ambientes restauradores ofrecen una diversidad de beneficios fisiológicos y psicológicos, pero hay pocos estudios que aborden en qué medida esta restauración ocurre para los niños; considerando el potencial que los ambientes restauradores podrían ejecutar, para colaborar con el desempeño o perfeccionamiento del funcionamiento de los niños, esa brecha es crítica (Bagot, Allen y Toukhsati, 2015). La utilización de ambientes que mejoran, es decir, que apoyan procesos restauradores y perfeccionan el desarrollo de los niños, así como aquellos con atención comprometida, serían beneficiosos y relevantes para las configuraciones educativas.

La ART apunta a que los ambientes naturales poseen mayor calidad física para la recuperación (Kaplan y Kaplan, 1989; Kaplan, 1995). Sin embargo, el agotamiento de la atención puede ser restaurado, y un lugar donde la fatiga mental puede ser restaurada es concebido, precisamente, como un ambiente restaurador (Kaplan, Stephen y Ryan, 1998). Los entornos restauradores incluyen una amplia variedad de configuraciones y de componentes naturales; en concreto, la vegetación y el agua parecen ser especialmente útiles para reducir la fatiga mental (Kaplan, Bardwell y Slakter, 1993; Kaplan, Stephen y Ryan, 1998). No existen estudios latinos sobre el efecto de la teoría de la restauración de la atención en espacios escolares. En este sentido, este artículo pretende presentar el conocimiento sistematizado obtenido en diferentes países y publicado en periódicos del área, exponiendo el estado del arte de este campo de estudio, y buscando fomentar nuevas investigaciones en el área. 


\begin{tabular}{|c|c|c|c|}
\hline Autores y año & Tipo de búsqueda & Perfil de muestra / ambiente & Objetivo \\
\hline Han (2009) & Casi-Experimental & $\begin{array}{l}\text { Estudiantes con una edad media } \\
\text { de } 13 \text { años }\end{array}$ & $\begin{array}{l}\text { Examina los efectos de las plantas } \\
\text { visibles en una sala de clase }\end{array}$ \\
\hline Rodney H. Matsuoka & Transversal & Escuela secundaria & $\begin{array}{c}\text { Si las características del paisaje del } \\
\text { campus tienen el impacto en el } \\
\text { comportamiento académico de los } \\
\text { alumnos }\end{array}$ \\
\hline Roe; Aspinall (2011) & Experimental & $\begin{array}{l}\text { Estudiantes de edad media de } \\
\qquad 11 \text { años }\end{array}$ & $\begin{array}{c}\text { Si las interacciones con la naturaleza } \\
\text { podrían mejorar los aspectos de la } \\
\text { cognición en los jóvenes }\end{array}$ \\
\hline Bagot, Allen, Toukhsati (2015) & Transversal & $\begin{array}{l}\text { Estudiantes con edad media de } \\
\qquad 10 \text { años }\end{array}$ & $\begin{array}{l}\text { Identificar los factores asociados a la } \\
\text { restauración percibida de los ambientes } \\
\text { de los niños }\end{array}$ \\
\hline Li, Sullivan (2016) & Experimental & $\begin{array}{l}\text { Estudiantes de entre } 14 \text { y } 16 \\
\text { años }\end{array}$ & $\begin{array}{l}\text { Investigar si las vistas verdes promueven } \\
\text { la restauración de la atención }\end{array}$ \\
\hline Akpinar (2016) & Transversal & $\begin{array}{c}\text { Estudiantes con edad entre } 12 \\
\text { y } 20 \text { años }\end{array}$ & $\begin{array}{l}\text { Determinar la asociación entre la } \\
\text { restauración percibida del verde del } \\
\text { ambiente escolar }\end{array}$ \\
\hline Kweon et al (2017) & Transversal & Escuela secundaria & $\begin{array}{c}\text { Este estudio examinó el efecto de los } \\
\text { visuales del aula y la relación con el } \\
\text { aprendizaje }\end{array}$ \\
\hline Hodson; Sander (2017) & Experimental & Escuela primaria & $\begin{array}{l}\text { Investigar la relación entre las } \\
\text { características ambientales urbanas y el } \\
\text { desempeño académico escolar }\end{array}$ \\
\hline
\end{tabular}

\begin{tabular}{|c|c|c|c|}
\hline Título & Autores & Año & Periódico \\
\hline $\begin{array}{l}1 \text { Influence of Limitedly Visible eafy Indoor Plants on the } \\
\text { Psychology, Behavior, and Health of Students at a Junior } \\
\text { High School in Taiwan }\end{array}$ & Ke-Tsung Han & 2009 & Environment and Behavior \\
\hline $\begin{array}{l}2 \text { Student performance and high school landscapes: } \\
\text { Examining the links }\end{array}$ & Rodney H. Matsuoka & 2010 & Landscape and Urban Planning \\
\hline $\begin{array}{l}3 \text { The restorative outcomes of forest school and } \\
\text { conventional school in Young people with good and } \\
\text { poor behavior }\end{array}$ & Jenny Roea, Peter Aspinallb & 2011 & $\begin{array}{l}\text { Urban Forestry \& Urban } \\
\text { Greening }\end{array}$ \\
\hline $\begin{array}{l}4 \text { Perceived restorativeness of children's school } \\
\text { playground environments: Nature, playground features } \\
\text { and play period experiences }\end{array}$ & $\begin{array}{l}\text { Kathleen L. Bagot, Felicity } \\
\text { Catherine Louise Allen, } \\
\text { SamiaToukhsati }\end{array}$ & 2015 & Psychology \\
\hline $\begin{array}{l}5 \text { Impact of views to school landscape son recovery from } \\
\text { stress and mental fatigue }\end{array}$ & $\begin{array}{l}\text { Dongying Li, William C. } \\
\text { Sullivan }\end{array}$ & 2016 & Landscape and Urban Planning \\
\hline $\begin{array}{l}6 \text { How is high school greenness related to students } \\
\text { restoration and health? }\end{array}$ & Abdullah Akpinar & 2016 & $\begin{array}{l}\text { Urban Forestry \& Urban } \\
\text { Greening }\end{array}$ \\
\hline $\begin{array}{l}7 \text { The link between school environments and student } \\
\text { academic performance }\end{array}$ & $\begin{array}{l}\text { Byoung-Suk Kweon, } \\
\text { Christopher D. Ellis, } \\
\text { JungaLee, Kim Jacobs }\end{array}$ & 2017 & $\begin{array}{l}\text { Urban Forestry \& Urban } \\
\text { Greening }\end{array}$ \\
\hline $\begin{array}{l}8 \text { Green urban landscapes and school-level academic } \\
\text { performance }\end{array}$ & $\begin{array}{l}\text { Cody B. Hodson, Heather A. } \\
\text { Sander }\end{array}$ & 2017 & Landscape and Urban Planning \\
\hline
\end{tabular}




\section{MÉTODO}

La revisión sistemática es un método que posibilita el potencial de una búsqueda, encontrando el mayor número de resultados posibles de forma ordenada (Costa y Zoltowski, 2014). De esta forma, se realizó una revisión sistemática de la literatura de los trabajos publicados en periódicos revisados por pares, a través del portal de Periódicos CAPES, conducida para resumir la investigación, y de una búsqueda de las palabras claves en el idioma inglés "attention restoration theory and school". La definición de las palabras clave, fue a través de la herramienta de búsqueda Psyclnfo, que tiene el objetivo de proporcionar la unificación de terminologías que se emplean en la comunidad científica, y son constantemente actualizadas por medio de nuevos descriptores. La muestra comprendió los siguientes criterios de inclusión: publicaciones de artículos indexados en periódicos y en el idioma inglés.

La revisión fue realizada mediante la búsqueda avanzada, con las palabras en: cualquiera: Attention restoration theory; y en el título: school. Así, el resultado final fue de 25 artículos, siendo 11 revisados por pares. Los estudios fueron seleccionados por medio de búsqueda electrónica en las siguientes bases de datos: Scopus (Elsevier) $\mathrm{N}=10$; Elsevier (Cross Ref) $\mathrm{N}=8$; (en el caso de las mujeres); Ciencias Sociales Citation Index (Web of Science) $\mathrm{N}=$ 8; Un archivo (GALE) $\mathrm{N}=6$; Science Citation Index Expanded (Web of Science) $N=6$; Materials Science \& Engineering Fecha base $=6$; Technology Research Fecha base $\mathrm{N}=5$; Civil Engineering Abstracts $\mathrm{N}=$ 5; Engineering Research Database N = 5; AGRIS (Organización de las Naciones Unidas, Organización de la Agricultura y la Agricultura) $\mathrm{N}=1$; ERIC (U.S Departamento de Educación) $\mathrm{N}=1$; SAGE Journals $\mathrm{BN}=1 ; \mathrm{N}=1$ y ASIA: Applied Social Science Index y Abstracts $N=1$. La estrategia de búsqueda en las bases de datos electrónicas incluyó investigaciones publicadas entre los años 2001 a 2017.

Después del análisis de los títulos, resúmenes y documentos completos, se seleccionó para el banco de datos final un total de 8 artículos científicos. La evaluación de los estudios dio énfasis en los resultados presentados, ya que la mayor parte de estos trabajos planteaba delineamientos experimentales; y la técnica empleada fue la revisión narrativa en profundidad de los artículos seleccionados (Tabla 1).

\section{DISCUSIÓN Y RESULTADOS}

Para obtener un panorama general de los estudios encontrados, se los presenta agrupados en la Tabla 2, compuesta por los siguientes ítems: autores, el tipo de investigación aplicado en el estudio, el perfil de la muestra y / o ambiente, el objetivo. Después, se realizó un análisis narrativo de dichos estudios.
Conforme a los programas de desarrollo de las Naciones Unidas (UNPD, 2015), las poblaciones de hoy habitan cada vez más en ambientes urbanos, lo que se equipara a la mitad de la población mundial; número que, además, crece día a día. Se prevé que hasta el 2050 , el $70 \%$ de la población convivirá en ambientes urbanos. Este cambio en el ambiente diario refleja la desconexión de las personas con la naturaleza, pero no sólo en los adultos: los niños también sufren la falta de contacto con el ambiente natural.

Las principales cuestiones planteadas sobre este tema se enfocan en la identificación de los lugares en que el ambiente natural influye en el desempeño de los alumnos, así como los elementos del paisaje y los tipos de exposición de la naturaleza. La búsqueda de resultados para estas problemáticas puede contribuir a comprender cómo ocurre esa relación, y a colaborar para beneficiar a los estudiantes a través de una arquitectura y un diseño que integren a la naturaleza (Hodson y Sander, 2017). Los citados autores buscaron identificar cuál es la asociación entre el desempeño académico y los niveles de vegetación en el ambiente escolar. Sus hallazgos indican que el aumento de la exposición a los espacios verdes como arboledas, está relacionado con la mejora del desempeño de los alumnos en pruebas de lectura; lo cual indica un beneficio en aras del perfeccionamiento académico.

La relación de niños y ambientes naturales es reconocida como necesaria, ya que ese contacto auxilia en su desarrollo cognitivo en la medida en que contribuye al bienestar mental y físico. La falta de este contacto se puede reflejar en el progreso de los niños, principalmente en lo que se refiere al desempeño escolar. Los diversos estudios revisados indican las ventajas del contacto con la naturaleza, como la mejora de la salud física (Kardan et al., 2015; Wheeler et al., 2015; White, et al., 2013), la salud mental (Bratman et al., 2015; Berman et al., 2012; Ryan et al., 2010) y la cognición, así como capacidades atencionales (Dadvand et al., 2015; Taylor y Kuo, 2009. Estos hallazgos también señalan que el tiempo en contacto con elementos naturales puede afectar positivamente resultados que se refieren a la educación. Además, el contacto, aunque solo sea visual, con la naturaleza, también puede contribuir a la recuperación de la fatiga mental, y como consecuencia a la salud (Han, 2009).

Según Bagot, Allen y Toukhsati (2015), las investigaciones con adultos revelan que los ambientes sin naturaleza también pueden considerarse como restauradores, por ejemplo, un museo (Kaplan, Bardwell y Slakter, 1993). Los factores contribuyentes para la restauración percibida en la ausencia de la naturaleza aún no han sido identificados en ambientes infantiles. Dos contribuyentes potenciales son otras características del patio y experiencias de tiempo de juego. Asimismo, en lo que se refiere a jóvenes, como proponen Roe y Aspinall (2011), los ambientes naturales tienden a favorecer aún más a aquellos con mal comportamiento, pues indica que la restauración puede variar dependiendo del estado de comportamiento del usuario. Es decir, indican que los ambientes na- 
turales pueden reducir la rabia y mejorar el humor, y sugieren que intervenciones en este ámbito son prometedoras para mejorar dicho comportamiento.

Para Li y Sullivan (2016) incluir en el cronograma de enseñanza un ambiente proyectado que incorpore el acceso y uso de espacios verdes, puede permitir que los alumnos recuperen sus capacidades de atención. En este estudio, los autores sugieren que 10 minutos de interacción con áreas verdes podrían ayudarlos a recuperarse de las actividades estresantes del ambiente escolar. Esta constituye una intervención de bajo costo que podría promover un efecto duradero sobre varias generaciones de estudiantes. Los arquitectos deben garantizar que las aulas tengan vistas visuales a los espacios verdes y, por tanto, deben considerar durante el proceso de proyecto la necesidad de implantar, efectivamente, vista a tales espacios. Estos recursos de paisaje pueden contribuir no sólo a lograr objetivos estéticos, sino también a que las intervenciones generen una ganancia de aprendizaje para alumnos y profesores.

Este argumento también se refuerza a través del estudio de Kweon et al. (2017), donde se sugiere que los árboles también pueden desempeñar un papel importante en los entornos de aprendizaje. Las escuelas con más árboles tuvieron mejor desempeño de los alumnos en pruebas de matemáticas y lectura, y esta relación también fue encontrada por Matsuoka (2010). Si los árboles son capaces de marcar una diferencia significativa en el desempeño escolar general, los ambientes escolares deben considerar los árboles en su infraestructura.

Además, los beneficios de la naturaleza no se restringen sólo en beneficios sobre el desempeño escolar, ya que la escuela no está compuesta sólo por aulas, hay otros ambientes que lo componen, y estos espacios también pueden interferir en los usuarios. Según el mismo Matsuoka (2010), ambientes donde los alumnos se alimentan, también necesitan de visuales con vegetación. El autor identificó, a través de su investigación con 101 escuelas de enseñanza media, que visuales con mayores cantidades de árboles y arbustos, están positivamente asociadas a los resultados de interés de los alumnos que planean ingresar en una universidad, así como, a un mejor comportamiento. En otras palabras, en aquellas escuelas que proyectan sus espacios con más áreas verdes, es más probable que los alumnos tengan menos comportamientos violentos, como por ejemplo, violencia verbal y física.

Para Akpinar (2016), cuyo trabajo fue realizado a través de una investigación transversal, estudios futuros deben considerar una medida más objetiva, pues, si bien se puede concluir que el espacio verde lleva a una mejora en la salud mental y física, existe una relación transversal y asociativa que debe analizarse. Efectivamente, los datos recolectados a través de testimonios presentan sólo parte del efecto restaurador del ambiente, por lo que es importante realizar investigaciones experimentales y extensas, que logren verificar desde una perspectiva más amplia el efecto restaurador en comportamientos, no sólo a partir de lo que es percibido por los participantes.

\section{CONCLUSIONES}

Las referencias seleccionadas para el desarrollo de este artículo confirmaron, por medio de esta investigación, la posibilidad de proporcionar exposición a los ambientes naturales para estudiantes, ya que las características de estos espacios pueden favorecer a una condición de vida saludable. Además, la relación entre los profesionales arquitectos con los educadores es fundamental para garantizar que estos ambientes sean adecuados, tanto desde el punto de vista físico, como cognitivo de los usuarios.

Diversos ámbitos de la investigación apuntan a reconectar niños, jóvenes y adultos a ambientes naturales. Mediante esta posible conexión, favorecida por el espacio escolar, se podría remediar algunos problemas asociados a la educación. Este estudio procuró ampliar el conocimiento sobre investigaciones en esta área, presentando propuestas que demostraron los efectos que la naturaleza puede promover tanto en jóvenes como en niños. Son necesarios futuros estudios aplicados en América del Sur para explorar cómo se desarrolla la restauración de la atención en espacios escolares, evaluando tanto los espacios externos como internos, con el fin de identificar determinadas formas de calificar el proceso de aprendizaje. 


\section{REFERENCIAS BIBLIOGRÁFICAS}

AKPINAR, Abdullah. How is quality of urban green spaces associated with physical activity and health? Urban Forestry y Urban Greening, 2016, vol. 16, pp. 76-83.

ARAÚJO, Alezandra. P. Q. C. Avaliação e manejo da criança com dificuldade escolar e distúrbio de atenção. Jornal de Pediatria, 2002, vol. 78, n 1, pp. 104-110.

BAGOT, Kathleen L.; ALLEN, Felicity Catherine Louise; TOUKHSATI, Samia. Perceived restorativeness of children's school playground environments: Nature, playground features and play period experiences. Journal of environmental psychology, 2015, vol. 41, pp. 1-9.

BERMAN, Marc G.; KROSS, Ethan; KRPAN, Katherine M.; ASKREN, Mary K.; BURSON, Aleah; DELDIN, Patricia J.; KAPLAN, Stephen; SHERDELL, Lindsey; GOTLIB, Ian H. y JONIDES, John. Interacting with nature improves cognition and affect for individuals with depression. Journal of affective disorders, 2012, vol. 140, n³, pp. 300-305.

BRATMAN Gregory N., HAMILTON J. Paul, HAHN Kevin S., DAILY Gretchen C., GROSS James J. Nature experience reduces rumination and subgenual prefrontal cortex activation. Proceedings of the national academy of sciences, 2015 , vol. $112, n^{\circ} 28$, pp. $8567-8572$.

CHOI, Hwan-Hee; VAN MERRIËNBOER, Jeroen JG y PAAS, Fred. Effects of the physical environment on cognitive load and learning: towards a new model of cognitive load. Educational Psychology Review, 2014, vol. 26, n² 2, p. 225-244.

COSTA, Angelo Brandeli y ZOLTOWSKI, Ana Paula Couto. Como escrever um artigo de revisão sistemática. Manual de produção científica, pp. 55-70, 2014

DADVAND, Payam; NIEUWENHUIJSENA, Mark J.; ESNAOLAA, Mikel; FORNSA, Joan; BASAGAÑAA, Xavier; PEDREROLA, M. Alvarez; RIVAS, loar; VICENTE, M. López; PASCUAL, M. De Castro; SUF, Jason; JERRETT, Michael; QUEROL, Xavier y SUNYER, Jordi. Green spaces and cognitive development in primary schoolchildren. Proceedings of the National Academy of Sciences, 2015, vol. 112, n²6, pp. 7937-7942.

HAN, Ke-Tsung. Influence of limitedly visible leafy indoor plants on the psychology, behavior, and health of students at a junior high school in Taiwan. Environmentand Behavior, 2009, vol. 41, n 5, pp. 658-692.

HARTIG, Terry. Restorative environments. En: C. Spielberger (ed.), Encyclopedia of applied psychology (vol. 3), pp. 273-279. Oxford: Academia, 2004.

HARTIG, Terry; MANG, Marlis; EVANS, Gary W. Restorative effects of natural environment experiences. Environment and behavior, v. 23, n. 1, p. 3-26, 1991.

HODSON, Cody B. y SANDER, Heather A. Green urban landscapes and school-level academic performance. Landscape and Urban Planning, 2017, vol. 160, pp. 16-27.

JAYARATNE, Kapila. Inculcating the ergonomic culture in developing countries: national healthy schoolbag initiative in Sri Lanka. Human factors, 2012, vol. 54, n 6, pp. 908-924.
KAPLAN, Rachel y KAPLAN, Stephen. The experience of nature: A psychological perspective. CUP Archive, 1989.

KAPLAN, Rachel; KAPLAN, Stephen y RYAN, Robert. With people in mind: Design and management of everyday nature. Island Press, 1998.

KAPLAN, Stephen. The restorative benefits of nature: Toward an integrative framework. Journal of environmental psychology, 1995, vol. 15, n³, pp. 169-182.

KAPLAN, Stephen; BARDWELL, Lisa V. y SLAKTER, Deborah $\mathrm{B}$. The museum as a restorative environment. Environment and Behavior, 1993, vol. 25, n 6, pp. 725-742.

KARDAN, Omid; GOZDYRA, Peter; MISIC, Bratislav; MOOLA, Faisal; PALMER, Lyle J.; PAUS, Tomáš y BERMAN, Marc G. . Neighborhood greenspace and health in a large urban center. Scientific Reports, 2015, vol. 5, pp. 11610.

KWEON, Byoung-Suk; ELLIS, Christopher D.; LEE, Junga y JACOBS, Kim. The link between school environments and student academic performance. Urban Forestry y Urban Greening, 2017, vol. 23, pp. 35-43

LI, Dongying y SULLIVAN, William C. Impact of views to school landscapes on recovery from stress and mental fatigue. Landscape and Urban Planning, 2016, vol. 148, pp. 149-158.

LØNDAL, Knut. Places for Child-Managed Bodily Play at an After-School Program. Children Youth and Environments, 2013, vol. 23, n², pp. 103-126.

MATSUOKA, Rodney $\mathrm{H}$. Student performance and high school landscapes: Examining the links. Landscape and urban planning, 2010, vol. 97, n 4, pp. 273-282.

ROE, Jenny y ASPINALL, Peter. The restorative outcomes of forest school and conventional school in young people with good and poor behaviour. Urban forestry \& urban greening, 2011, vol. 10, n³, pp. 205-212.

RYAN, Richard M.; WEINSTEIN, Netta; BERNSTEIN, Jessey BROWN, Kirk W.; MISTRETTA, Louis y GAGNE, Marylene. Vitalizing effects of being outdoors and in nature. Journa of Environmental Psychology, 2010, vol. 30, n² 2, pp. 159168.

TAYLOR, Andrea y KUO, Frances E. Children with attention deficits concentrate better after walk in the park. Journal of attention disorders, 2009, vol. 12, n 5, pp. 402-409.

UNPD. World urbanization prospects: The 2012 revision. New York: United Nations Department of Economic y Social Affairs, 2012

WHEELER, Benedict W.; LOVELL, Rebecca; HIGGINS, Sahran L.; WHITE, Mathew P.; ALCOCK, Ian; OSBORNE, Nicholas J.; HUSK, Kerryn; SABEL, Clive E. y DEPLEDGE, Michael $\mathrm{H}$. Beyond greenspace: an ecological study of population general health and indicators of natural environment type and quality. International journal of health geographics, 2015, vol. 14, n 1, p. 17.

WHITE, Mathew P. et al. Feelings of restoration from recent nature visits. Journal of Environmental Psychology, 2013, vol. 35, pp. 40-51. 